\title{
Comparison of outcomes between simultaneous fat grafting and filler injection with transconjunctival fat removal for improvement of the lower eyelid contour
}

Bong Moo Lee ${ }^{1}$, Joo Hyuk Park', Dae Hwan Park², Jeong Su Shim², Yong Jig Lee ${ }^{2}$, Seung Eun Kim²

${ }^{1}$ Lift Plastic Surgery Clinic, Daegu;

${ }^{2}$ Department of Plastic and

Reconstructive Surgery, Catholic

University of Daegu School of Medicine, Daegu, Korea
Background Mid-facial aging is associated with various changes, particularly around the lower eyelid, where these changes manifest as herniation of orbital fat, sagging of soft tissue around the zygoma, and the tear trough deformity. We improved the lower eyelid contour via transconjunctival orbital fat removal and the use of either an autologous fat graft or filler injection.

Methods Between June 2016 and July 2018, 100 patients aged 23-48 years underwent autologous fat grafts (group A: 50 patients, 100 eyes) or filler injections (group B: 50 patients, 100 eyes). The mean follow-up period was approximately 3 months. The results of surgery were evaluated using a visual analog scale (VAS) to assess patient satisfaction and with preoperative and postoperative scores determined by three plastic surgeons according to the grading system for lower eyelid contour deformity devised by Barton et al.

Results The mean VAS score was 8.48 in group A and 8.92 in group $\mathrm{B}$, but the difference was not statistically significant. The percentage of postoperative improvement from preoperative grade I deformity to grade o was $97.7 \%$ in group A and $100 \%$ in group B. The percentage of improvement from preoperative grade II deformity to grade 0 was $96.0 \%$ in group $A$ and $96.1 \%$ in group $B$, and the percentage of improvement from grade III deformity to grade 0 in was $66.7 \%$ in group $A$ and $75 \%$ in group $B$.

Conclusions Filler injection can be an alternative to fat grafting for improvement of the lower eyelid contour after transconjunctival orbital fat removal.

Keywords Eyelids / Cosmetic techniques / Skin aging / Hyaluronic acid

\section{INTRODUCTION}

Aging of the face is accompanied by various changes, especially in the mid-facial area. These changes include sagging of the soft tissue

Received: Mar 20, 2020 Revised: Apr 3, 2020 Accepted: Apr 6, 2020 Correspondence: Dae Hwan Park Department of Plastic and Reconstructive Surgery, Catholic University of Daegu School of Medicine, 33 Duryugongwonro 17-gil, Nam-gu, Daegu 42472, Korea

Tel: +82-53-650-4578, Fax: +82-53-650-4584, E-mail: dhpark@cu.ac.kr

Copyright @ 2020 The Korean Society for Aesthetic Plastic Surgery.

This is an Open Access article distributed under the terms of the Creative Commons Attribution Non-Commercial License (https://creativecommons.org/licenses/by-nc/4.0/) which permits unrestricted non-commercial use, distribution, and reproduction in any medium, provided the original work is properly cited. $\quad w w w . e-a a p s . o r g$ around the maxilla, skeletonization of the lower eye margin, palpebral bag formation, and development of the tear trough deformity $[1,2]$. These changes tend to result in a more tired and older appearance, which may precipitate the desire for surgery to improve the contour of the lower eyelid.

In patients with mild deterioration due to aging of the lower eyelid, corrective surgery may include transconjunctival fat reposition and septal reset. As a recent alternative to this approach, transconjunctival fat removal has been performed. Transconjunctival fat repositioning involves a longer operation time than removal and is relatively inconvenient for patients due to the need for postoperative suture removal. Furthermore, reoperation may be required if the repositioned fat does not remain in place after surgery. In transconjunctival fat removal, after removal of the lower eyelid bag 
fat, an insufficient volume of fat remains, necessitating refilling of the lower eyelid. Two methods for this refilling are autologous fat grafts and filler injections. In this study, we compared these two methods.

\section{METHODS}

We retrospectively reviewed the medical records of patients who underwent transconjunctival fat removal to correct the contour of the lower eyelid between June 2016 and July 2018. Patients who underwent this procedure with additional autologous fat grafting or filler injection were included. Those with an infectious disease or history of mental illness that could affect wound recovery or the outcome of the procedure were excluded. Patients whose skin and eyelid muscles displayed severe bags (requiring lower eyelid surgery involving manipulation of the skin and muscles) as well as those with congenital malformations or acquired trauma, burns, or eyelid or eye diseases were also excluded from this study. The average follow-up time of the included patients was 3 months, and the medical records reviewed included patient age, clinical photographs, surgical records, and information regarding complications.

A total of 50 randomly selected patients who underwent autologous fat grafting after transconjunctival fat removal were classified as group $\mathrm{A}$, and 50 randomly selected patients who underwent filler injection after transconjunctival fat removal were assigned to group B. The procedures employed were as follows.

First, propofol was administered as preoperative anesthesia; then, operative local anesthesia was administered using a solution of $2 \%$ lidocaine and epinephrine at a ratio of 1:100,000 (2\% lidocaine $\mathrm{HCl}$ and 1:50,000 epinephrine). Ten minutes after administration of local anesthesia, the conjunctiva was incised with a Bovie electrosurgical unit (Bovie Medical Corp., Melville, NY, USA) and then dissected using a preseptal approach. The incision was made at the orbital septum to properly remove the orbital fat in the medial, central, and lateral areas; then, the patient was seated for confirmation of proper fat removal and symmetry. After this confirmation, the injured orbital septum was repaired with 7-0 sutures (Surgifit; Ailee Co. Ltd., Busan, Korea). The operation was completed after sufficient hemostasis.

In the cases in which autologous fat grafting was performed, the fat removal for grafting was done before the removal of fat from the lower eyelid. While in the prone position, the patient was injected with tumescent solution ( $300 \mathrm{~mL}$ of saline $+20 \mathrm{~mL}$ of $2 \%$ lidocaine $+1 \mathrm{~mL}$ of epinephrine mixed solution) into the predesignated donor areas, which were on the inside and outside of the thigh. Fat was collected using prepared cannulae with 1-mm and $0.5-\mathrm{mm}$ diameter holes and prepared by centrifugation for $1 \mathrm{~min}-$ ute at $3,200 \mathrm{rpm}$.

For fat injection, the fat collected with the 1-mm cannula was injected to fill the volume deficiency in the preperiosteal fat, and the fat obtained with the $0.5-\mathrm{mm}$ cannula was injected to refine the shallow layer. A 21-gauge blunt cannula was used for fat injection. The fat remaining after infusion was disposed of without storage.

For filler injection, Neuramis Lidocaine (Medytox, Seoul, Korea) was used to fill the volume in the deeper layer (the preperiosteal fat), while Neuramis Light Lidocaine (Medytox) was used to refine the shallower layer, below the orbicularis oculi. A 27-gauge blunt cannula was used for filler injection (Fig. 1).

Many studies have cited the grading system developed by Barton et al. to evaluate the degree of deformation of the lower eyelid [3]. Similarly, in this study, the Barton grading system was used to evaluate the clinical results of surgery. Under this system, grade 0 is defined as the absence of medial or lateral lines demarcating the arcus marginalis or the orbital rim and a smooth youthful contour without a transition zone at the orbit-cheek junction. Grade I involves the mild, subtle presence of a medial line or shadow and a smooth lateral transition of the lid-cheek junction. Grade II indicates moderate prominence of a visible demarcation of the lid-cheek junction that extends from the medial to the lateral side, and grade III indicates severe demarcation of the orbit-cheek junction with an obvious step between the orbit and the cheek.

The classification of the results according to this grading system was performed by three plastic surgeons (DHP, BML, JHP) based on patients' medical records and clinical photographs. After an initial classification of the eyes in each group, the degree of lower eyelid deformation was again evaluated in the same fashion after surgery in order to assess improvement.

In this study, subjective patient satisfaction was evaluated at 3

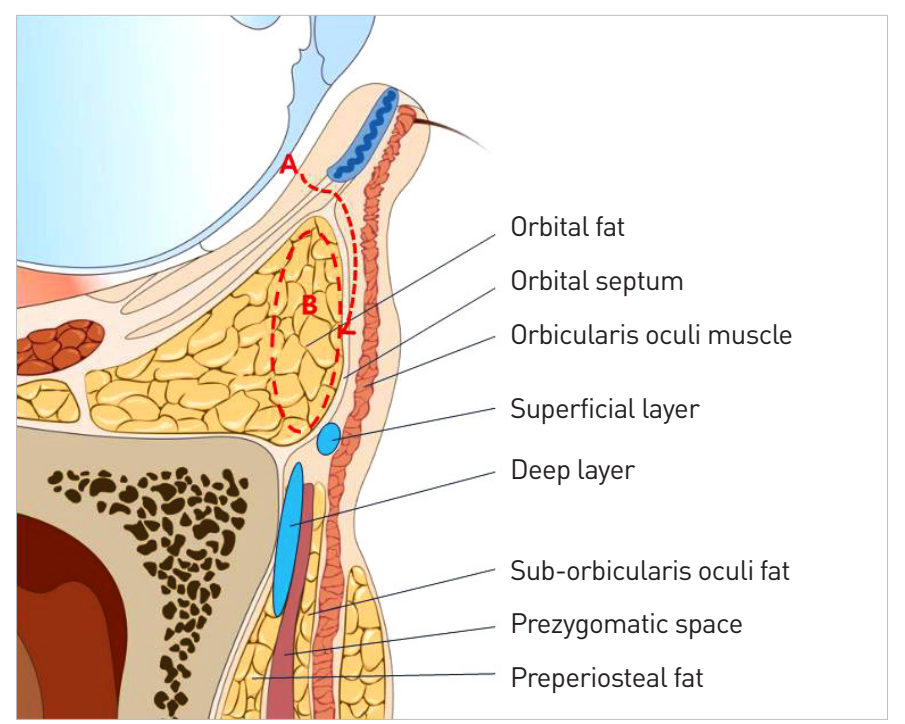

Fig. 1. After incision at the conjunctiva, the orbital preseptal plane was exfoliated to approach (A: approaching course to orbital fat) orbital fat (B: orbital fat to be removed); after fat removal, filler injection was done at deep layer (preperiosteal fat) and superficial layer (below orbicularis oculi muscle). 
months after surgery using a visual analog scale (VAS) ranging from 1 point (not satisfied) to 10 points (completely satisfied) [4]. Results were analyzed using the Fisher exact two-tailed test for grade I eyes and the Wilcoxon rank-sum test for eyes of grades II and III.

\section{RESULTS}

The average age of the patients was 36.4 years (range, 23-46 years) in group A and 38.6 years (range, 25-48 years) in group B. In the preoperative assessment performed according to the Barton grading system, grades I, II, and III were assigned to 44,50 , and six eyes in group A and 41, 51, and eight eyes in group B, respectively.

In group $\mathrm{A}$, of the eyes categorized preoperatively as grade I, 43 (97.7\%) were categorized postoperatively as grade 0 , while one (2.3\%) was categorized as grade I. Of the eyes categorized preoperatively as grade II, $48(96.0 \%)$ were determined postoperatively to be grade 0 , while two (4.0\%) were determined to be grade I. The eyes categorized preoperatively as grade III were categorized postoperatively as grade 0 (four eyes, 66.7\%) and grade I (two eyes, 33.3\%) (Tables 1,2).

In group B, all 41 of the eyes (100\%) categorized preoperatively as grade I were categorized postoperatively as grade 0 . Of the eyes categorized preoperatively as grade II, 49 (96.1\%) were determined postoperatively to be grade 0 , while two (3.9\%) were determined to

Table 1. Preoperative comparison between group A lautologous fat graft) and group B (filler injection)

\begin{tabular}{llccc}
\hline \multirow{2}{*}{ Group } & \multicolumn{3}{c}{ Preoperative Barton grade, No. (\%) } & \multirow{2}{*}{ Total No. (\%) } \\
\cline { 2 - 3 } & Grade I & Grade II & Grade III & \\
\hline A & $44(44)$ & $50(50)$ & $6(6)$ & $100(100)$ \\
B & $41(41)$ & $51(51)$ & $8(8)$ & $100(100)$ \\
\hline
\end{tabular}

a) $P>0.05$

Table 2. Postoperative comparison between group A lautologous fat graft) and group B (filler injection)

\begin{tabular}{lccccc}
\hline \multirow{2}{*}{ Group } & \multicolumn{3}{c}{ Postoperative Barton grade, No. (\%) } & \multirow{2}{*}{$\begin{array}{c}\text { Total No. } \\
\text { (\%) }\end{array}$} \\
\cline { 2 - 5 } & Grade 0 & Grade I & Grade II & Grade III & \\
\hline A & $95(95)$ & $5(5)$ & 0 & 0 & $100(100)$ \\
B & $96(96)$ & $2(2)$ & 0 & 0 & $100(100)$ \\
\hline
\end{tabular}

al) $>0.05$.

Table 3. VAS score of patient satisfaction (3 months after surgery)

\begin{tabular}{lc}
\hline Group & Mean VAS score \\
\hline A (autologous fat graft) & 8.48 \\
B (filler injection) & 8.92 \\
\hline
\end{tabular}

VAS, visual analog scale. be grade I. Finally, of the eyes categorized preoperatively as grade III, six (75.0\%) and two (25.0\%) eyes were categorized postoperatively as grades 0 and I, respectively (Tables 1,2 ).

For patient satisfaction, the VAS score was 8.48 (of 10) in group $A$ and 8.92 in group $B$, although this difference was not statistically significant $(\mathrm{P}>0.05)$ (Table 3$)$.

\section{Case 1}

A 31-year-old woman underwent transconjunctival fat removal and fat autografting on both sides after complaining of bilateral lower eyelid contour deformation. Before surgery, both lower eyelids were grade I, and fat autografts of $2.0 \mathrm{~mL}$ and $2.2 \mathrm{~mL}$ were injected into the left and right eyelid, respectively. A comparison with the preoperative photograph showed clear improvement of the lower eyelid contour 3 months after surgery (Fig. 2).

\section{Case 2}

A 28-year-old woman underwent transconjunctival fat removal and filler injection on both sides after complaining of bilateral lower eyelid deformation. The patient's left lower eyelid was determined to be grade II, while her right lower eyelid was grade I. After surgery, $1.4 \mathrm{~mL}$ and $1.0 \mathrm{~mL}$ of filler were injected into the left and right eyelid, respectively. A comparison with the preoperative photograph showed clear improvement and increased symmetry of the lower eyelid contour 3 months after surgery (Fig. 3).

\section{Case 3}

A 24-year-old woman underwent transconjunctival fat removal and fat autografting on both sides after complaining of bilateral lower eyelid deformation. Before surgery, both lower eyelid contours were grade I, and after surgery, $3.2 \mathrm{~mL}$ and $2.9 \mathrm{~mL}$ of fat were injected into the left and right sides, respectively. A comparison
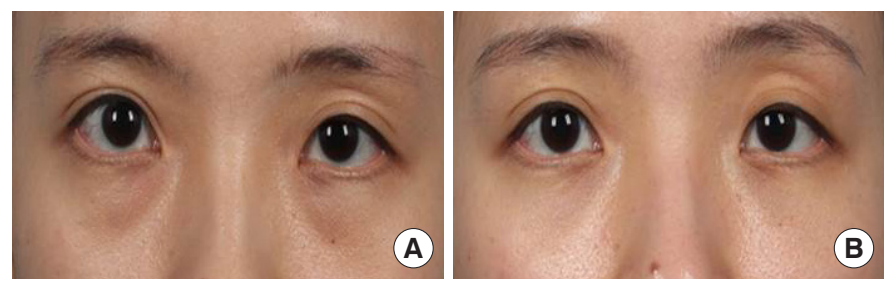

Fig. 2. Case 1. (A) Before and (B) 3 months after the operation.
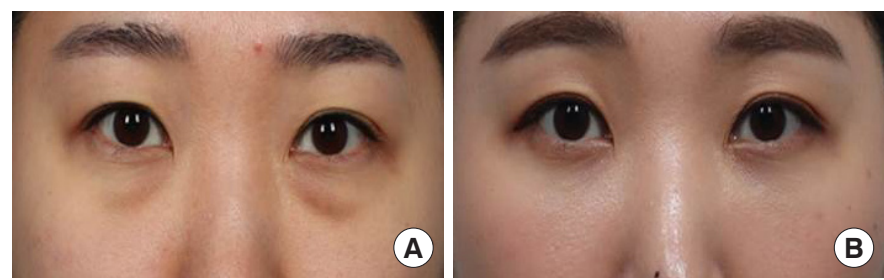

Fig. 3. Case 2. (A) Before and (B) 3 months after the operation. 

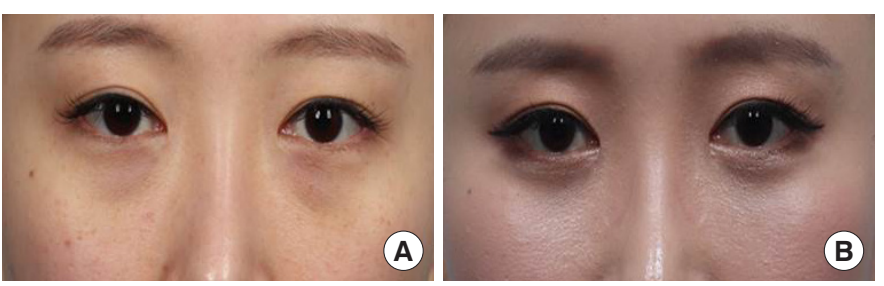

Fig. 4. Case 3. (A) Before and (B) 3 months after the operation.

with the preoperative photograph showed clear improvement of the lower eyelid contour 3 months after surgery (Fig. 4).

\section{Case 4}

A 36-year-old man underwent transconjunctival fat removal and filler injection on both sides after complaining of bilateral lower eyelid deformation. Before surgery, both lower eyelid contours were grade II, and after surgery, $0.8 \mathrm{~mL}$ and $0.7 \mathrm{~mL}$ of filler were injected into the left and right sides, respectively. A comparison with the preoperative photograph showed clear improvement of the lower eyelid contour 3 months after surgery (Fig. 5).

\section{DISCUSSION}

The face ages just like any other body part, and the lower eyelids in particular stand out in this regard. According to Mendelson [5], the aging of the central face is due to the loss of elasticity and subsequent stretching of soft tissue, changes in the orbicularis oculi muscle, and weakened support of the orbital fat, orbital septum, and canthus. The orbital fat thus protrudes forward from the lower eye socket, and the orbicularis oculi muscle slowly descends. Finally, the once flat lower eyelid bulges, resulting in changes to the outline of the lower eyelid, such as palpebral bag and tear trough deformity development.

Many beauty procedures and operations are used to correct these types of lower eyelid outline changes. These vary from simple procedures, such as filler injections, the usage of high-intensity focused ultrasound equipment, fat autotransplantation, and thread injection to surgery, including lower orbital fat extirpation, fat relocation, and palpebromalar enhancement using prosthetics. Lately, many patients have begun to prefer simple microinvasive procedures, as they have fewer side effects and faster recovery periods. Although they do improve lower eyelid outlines to some extent, these procedures have a limited effect on heavily impacted outlines, in which case surgery becomes preferable. A surgical approach allows the plastic surgeon to operate on drooping skin, orbicularis oculi muscle, and orbital fat invasively and aggressively in order to improve lower eyelid contour; however, postoperative pain, delayed return to everyday life due to swelling, and scarring may occur. Therefore, for effective correction of lower eyelid contour deformation, it is important to weigh the advantages and disadvan-
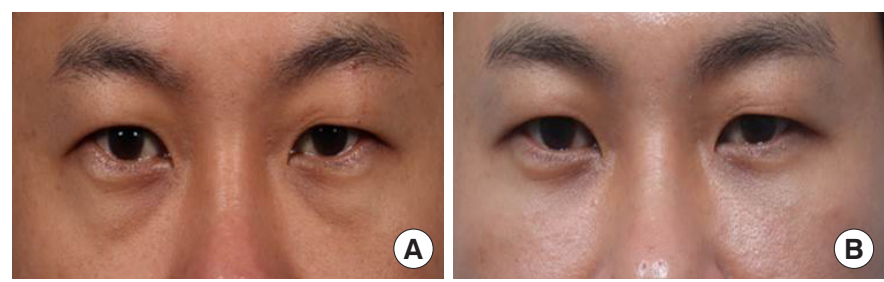

Fig. 5. Case 4. (A) Before and (B) 3 months after the operation.

tages of noninvasive procedures and invasive surgery.

Early fat relocation surgery has the downsides of longer surgery time, discomfort due to the removal of sutures needed to fix the relocated fat after surgery, and a possible need for revision surgery if the relocated fat is not maintained after suture removal (compared to lower orbital fat extirpation) [6]. There is also a possibility of necrosis, calcification of the relocated fat, and (according to the literature) discomfort due to pulling of the lower eyelid, with diplopia in severe cases [7]. In addition, due to the limited fat volume used for relocation, additional fat is needed after relocation to correct for any lack of the palpebromalar groove and mid-facial volume. Therefore, as an alternative to fat relocation surgery and especially due to the fast surgical and recovery time, fat autografting or filler injections for volume replacement after lower orbital fat extirpation have begun to be used.

Although bulging of the lower eyelid can be improved by removing orbital fat using lower orbital fat extirpation, areas of insufficient fat volume must be filled below the infraorbital rim and at the palpebromalar groove, including the tear trough deformity, for enhanced contour improvement and patient satisfaction. Fat autografting is used for this purpose. Fat survival differences among patients make it difficult to predict outcomes precisely, and those with lower engraftment figures require reoperation; however, relatively little fat is necessary to fill the volume defect. For these reasons, filler injection after lower orbital fat extirpation may be considered as an alternative to fat autografting. Thus, these techniques were compared in this study using patients who had undergone the different procedures.

After selecting patients and separating those who had undergone fat autografting after transconjunctival fat removal from those who had been treated with filler injection, the procedural steps were divided. The grading scale proposed by Barton et al. [2] was employed; this scale uses clinical before-and-after photographs to divide eyelids into four grades, from grade 0 (no deformation) to grade III (severe deformation). The proportions of patients who displayed improvement at each grade were calculated after surgery to evaluate the clinical effect, and the patients were surveyed regarding satisfaction with the results.

The results showed that for grades I, II, and III, group A demonstrated a reduction in grade in $97.7 \%, 100 \%$, and $100 \%$ of cases, and group B showed improvement in 100\%, 100\%, and 100\% of 
cases respectively. Group B thus had a slightly higher percentage of success, but the difference was not significant. Patient satisfaction was evaluated using VAS scores. Group A had a mean score of 8.48 points (out of 10), while group B had a mean score of 8.92. Again, group B scored higher than group A, but not significantly so. Thus, filler injection was determined to be as effective as fat autotransplatation after transconjunctival fat removal. The slightly higher patient satisfaction score recorded for group B could have been due to the clinical effect (i.e., improvement of the lower eyelid contour), but it could also have been related to reduced discomfort after surgery and the shortened recovery time.

In the revision of lower eyelid deformities, patients prefer fat autografting to prosthesis implantation or injection because it uses their own tissue. However, the survival rate of refined centrifuged fat tissue is different for each patient, which makes it difficult to predict the exact outcome of fat autografts. Furthermore, according to previous research, there is a tendency to lose $30 \%-83 \%$ of the total volume after the procedure [8]. This means that, although there may be some differences among studies, some degree of fat tissue loss after fat autografting is likely. Therefore, a disadvantage of this procedure is that the patient must endure an unnatural cosmetic look until the targeted contour appears after the natural volume loss post-surgery. This is due to the overcorrection needed to eventually yield a natural lower eyelid contour when carrying out a fat autograft.

Additionally, as mentioned, secondary surgery may be needed when the lower eyelid contour does not meet the expected outcome, since the engraftment volume and fat volume loss rate differ among patients. This is associated with the inconvenience of having to extract the fat tissue again, since studies have shown that due to the decreased survival rate of the frozen centrifuged fat tissue, it is difficult to store it after surgery [9]. Also, side effects, such as infection leading to flare and ache, could occur when injecting fridgekept fat, causing potential discomfort and risk to the patient [10].

However, according to the literature, volume loss after filler injection does not occur until the sixth month after the procedure. This carries the advantage of reduced discomfort resulting from corrective surgery, and even when there is a need of retreatment, simple correction can be achieved. However, after the sixth month after the procedure, we must consider the longevity of the grafted fat and filler. In contrast, after autologous fat is taken, it can survive for a longer period than can filler.

Furthermore, the eyelids preoperatively categorized as grade III displayed improvement to grade I at proportions of $33.3 \%$ and $25 \%$ in groups A and B, respectively. Preoperative grade III eyelids exhibited a lower rate of improvement to grade 0 than did preoperative grade I and II eyelids. Since patients with severe (grade III) lower eyelid contour deformation have heavily aged structures, their eyelids cannot be corrected using relatively simple transconjunctival fat removal. Thus, these patients must receive lower eyelid plastic surgery or lifting of the central face region to correct droop- ing of the skin and orbicularis oculi muscle [11].

In this study, it was relatively difficult to compare the results with those of control patients who underwent fat removal only. This was because only a few cases displayed clinically satisfactory results from surgery alone, and those patients still wanted to correct the insufficient volume at the periphery. As an additional limitation, only one subjective grading scale and one evaluation of satisfaction were used; thus, this study lacked objective evaluation indexes. When additional research methods allow us to measure three-dimensional volumes using patient progress pictures to objectively evaluate the recovered volume and the degree of volume maintenance, and when further studies have been conducted to compare the survival rates of grafted fat under the eye with filler maintenance periods, more reliable conclusions will be possible.

In summary, both transconjunctival fat removal and filler injection are used to improve lower eyelid contour in patients with agerelated deformity. In this study, fat autografting and filler injection were compared as ways to provide the needed volume after transconjunctival fat removal. No significant differences between the two methods were found. However, considering that fat autografting is associated with the discomfort of retreatment due to survival rate differences, as well as with longer surgery time and the need for additional fat extraction when retreatment is necessary, filler injections appear to be a promising alternative for providing volume after transconjunctival fat removal.

\section{NOTES}

\section{Conflict of interest}

No potential conflict of interest relevant to this article was reported.

\section{Ethical approval}

The study was approved by the Institutional Review Board of Daegu Catholic University Medical Center (IRB No. CR-20-079) and performed in accordance with the principles of the Declaration of Helsinki.

\section{Patient consent}

The patients provided written informed consent for the publication and the use of their images.

\section{ORCID}

Bong Moo Lee Joo Hyuk Park Dae Hwan Park Jeong Su Shim Yong Jig Lee Sung-Eun Kim

https://orcid.org/0000-0001-8129-7376 https://orcid.org/0000-0002-2482-8131 https://orcid.org/0000-0001-9671-6510 https://orcid.org/0000-0002-8458-4415 https://orcid.org/0000-0002-6470-5750 https://orcid.org/0000-0002-3413-7094 


\section{REFERENCES}

1. de la Plaza R, Arroyo JM. A new technique for the treatment of palpebral bags. Plast Reconstr Surg 1988;81:677-87.

2. Barton FE Jr, Ha R, Awada M. Fat extrusion and septal reset in patients with the tear trough triad: a critical appraisal. Plast Reconstr Surg 2004; 113:2115-21.

3. Castanares S. Blepharoplasty for herniated intraorbital fat; anatomical basis for a new approach. Plast Reconstr Surg (1946) 1951;8:46-58.

4. Mohadjer Y, Holds JB. Cosmetic lower eyelid blepharoplasty with fat repositioning via intra-SOOF dissection: surgical technique and initial outcomes. Ophthalmic Plast Reconstr Surg 2006;22:409-13.

5. Mendelson BC. Herniated fat and the orbital septum of the lower lid. Clin Plast Surg 1993;20:323-30.

6. Nassif PS. Lower blepharoplasty: transconjunctival fat repositioning.
Facial Plast Surg Clin North Am 2005;13:553-9.

7. Momosawa A, Kurita M, Ozaki M, et al. Transconjunctival orbital fat repositioning for tear trough deformity in young Asians. Aesthet Surg J 2008;28:265-71.

8. Yu NZ, Huang JZ, Zhang $\mathrm{H}$, et al. A systemic review of autologous fat grafting survival rate and related severe complications. Chin Med J (Engl) 2015;128:1245-51.

9. Kim YK, Park HS, Lee HJ. Studies on the proper storage period and change of -20 degrees C cryopreserved adipocyte. J Korean Soc Aesthetic Plast Surg 2006;12:33-42.

10. Maamari RN, Massry GG, Holds JB. Complications associated with fat grafting to the lower eyelid. Facial Plast Surg Clin North Am 2019; 27:435-41.

11. Nahai F. The art of aesthetic surgery: principles and techniques. Boca Raton: CRC Press; 2010. 